\section{A Mobile Handheld Computing Application for Recording Hand Hygiene Observations}

To the Editor-Currently, most hand-hygiene observations are performed by human observers. ${ }^{1}$ Data from these observations are frequently recorded on paper forms requiring a data entry step prior to the distribution of results. This delays the interpretation of results, increases the cost of data collection, and may introduce transcription errors.

Recently, other groups have developed computer-based electronic recording systems; some of these also use hand-held devices. ${ }^{2}$ However, systems designed for individual healthcare systems may not be easy to disseminate. To increase the availability of direct electronic recording of observations, we developed an interactive iPhone application and made it available to the public free of charge for use by anyone with an iPhone or iPod touch device.

We developed our iPhone application, iScrub 1.0, using Apple's Cocoa framework, the Objective-C programming language, and the XCode integrated development environment. Our application takes advantage of the iPhone's touch screen interface and is easily customized to reflect each user's own healthcare facility. Customization features allow users to organize recorded observations by specific units or clinics in their facility. During use, observers simply indicate when they arrive and leave a specific location from a list of menu-driven choices based on their institution. Users can also specify which job classifications they wish to record for each observation (eg, nurse, nurse's aid, physician, X-ray technician, etc). To record observations, users record whether healthcare workers have taken advantage of hand-hygiene opportunities before entering and after leaving patient rooms. iScrub, version 1.0, does not measure all of the World Health Organization's Five Hand Hygiene Moments of Opportunity.

Observers can also collect similar information for gowning, gloving, and the use of face masks or N95 respirators. The application is programmed to know what protective equipment is required for contact, droplet, and airborne isolation. Each entry is automatically time and date stamped, and a built-in confirmation step minimizes data entry errors. If mistakes are made, they can easily be erased with a "discard" button. All data are recorded and analyzed in real time locally in a database on the observer's device. Given WiFi access, the observer can export data (in comma-separated-value format) to an E-mail address of the user's choice. The data can be E-mailed after observations are completed.

To test the usability of iScrub, we had 23 infection control professionals share 11 iPod touches with the application installed to determine whether they could use the application.
Next, to determine the usability in a clinical setting, we performed a small pilot study to test both the usability and recording accuracy of iScrub. Two different observers recorded hand-hygiene opportunities in the medical intensive care unit at the University of Iowa Hospitals and Clinics (Iowa City, Iowa). One observer recorded the observations using a paper form, and the other used the iScrub application installed on an iPod touch device. The dual observation period lasted for a period of 6 days (averaging 2 hours per day: the unit's current observation period). We used a Wilcoxon signed rank test to detect potential differences in count data (ie, hand-hygiene observations) generated by the 2 methods. Note that, for this statistical test, we considered the human observer to represent "ground truth," and the results of the Wilcoxon signed rank test compared the counts obtained each day in the following observed categories: physicians, nurses, other, and off-unit consultants.

During both the first and second pilot sessions, the participating infection control professionals were comfortable using the interface within a few minutes of picking up the device. During our clinical pilot, our application proved easy to use, and we could not detect a significant difference between the iScrub data and the data that were generated concurrently by traditional methods. Users were able to send data collected by the application via E-mail to either one or multiple parties. Although other groups have developed electronic data entry systems, ours is based on a very popular mobile computing device platform. As of January 2010, Apple has sold 50 million iPhone and iPod touch devices, and users have downloaded more than 3 billion applications. ${ }^{3}$

iScrub is easy to download and use and can be modified to fit an individual institution's needs (Figure 1), thus increasing the potential for dissemination of electronically collected data. Unlike other recording methods, iScrub can be used in an inconspicuous fashion and thus diminish the potential effects of observers on healthcare worker behavior (eg, the Hawthorne effect). ${ }^{4}$

Although the program is free, it does require users to have or purchase an Apple mobile computing device. However, the investment of less than $\$ 200$ for an iPod touch device should be easy to offset with the labor saved by reducing data entry efforts. Note that although an iPhone requires a phone contract, an iPod touch does not and thus has a much lower total cost of ownership. Another limitation is that iScrub requires access to a $\mathrm{WiFi}$ connection.

iScrub, version 1.0, does not measure all of the World Health Organization's Five Hand Hygiene Moments of Opportunity. Future directions include allowing users to observe all of the 5 moments ${ }^{5}$ instead of just "in-room" and "outof-room" moments. iScrub, version 2.0, will also include a 

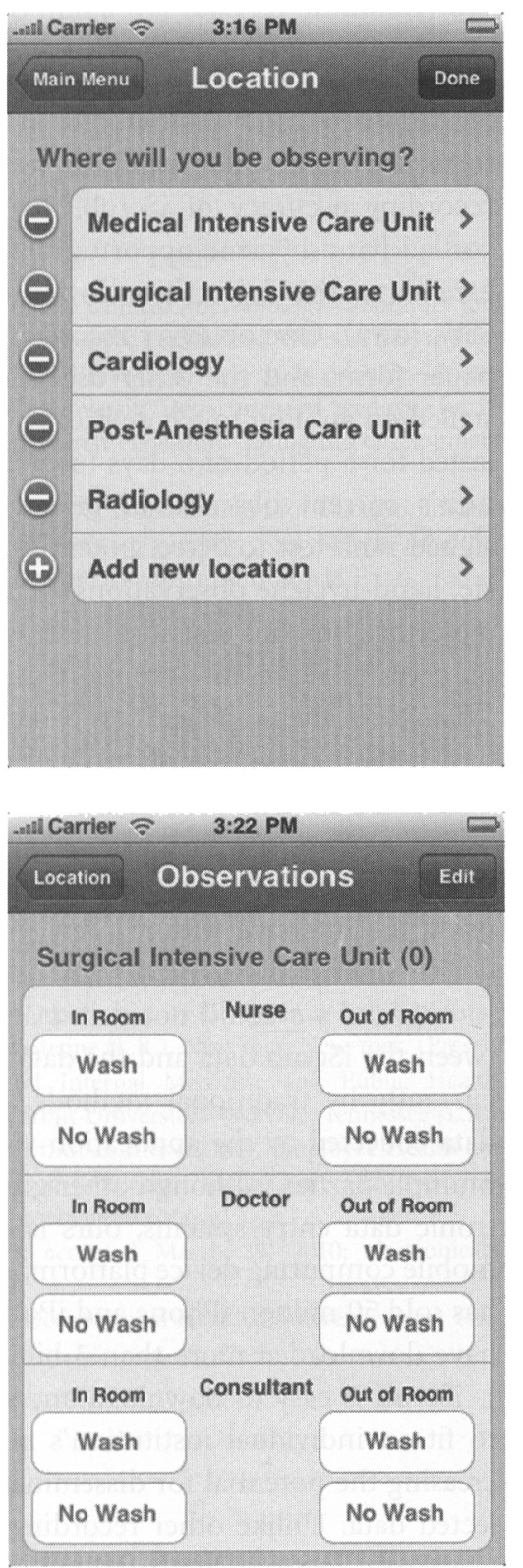
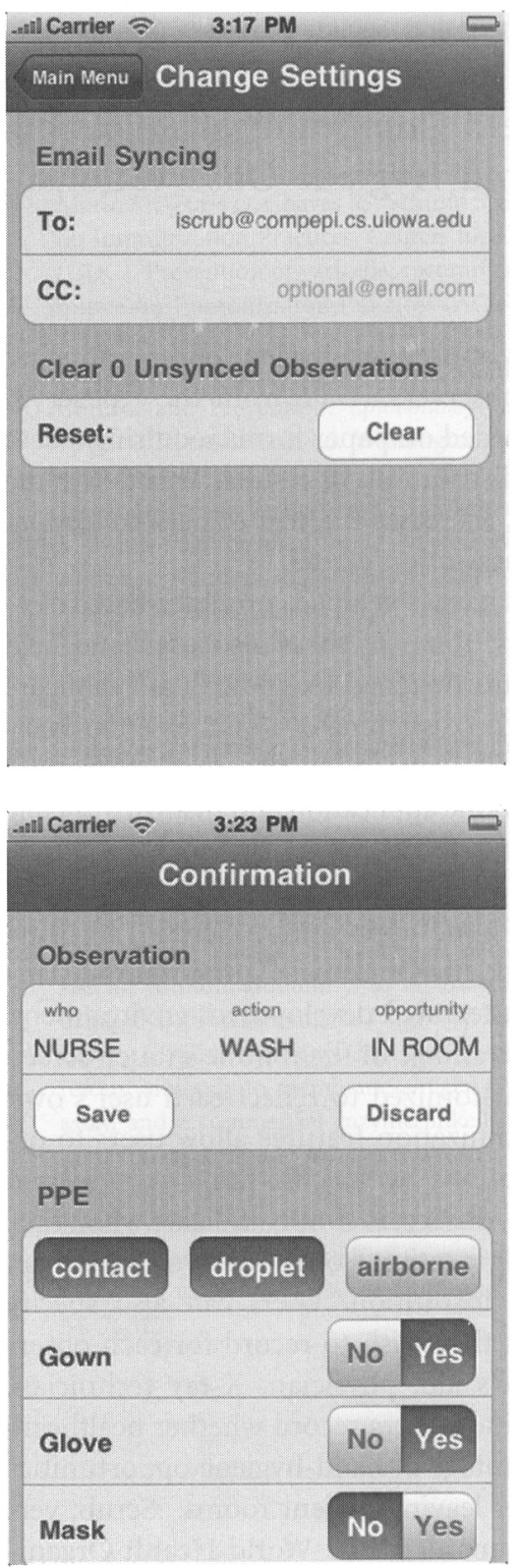

FIGURE 1. Application customization and data entry options (version 1.0).

secure companion Web site to allow the centralized aggregation and feedback of observations. Development of iScrub, version 2.0 , is in the beta phase, and its Web site will be able to scale to include hundreds of institutions or more. Such a system could support widespread (ie, state-wide) processimprovement projects and help to standardize the collection and reporting of hand-hygiene observations.

\section{ACKNOWLEDGMENTS}

Financial support. Support for this research was provided by the Centers for Disease Control and Prevention, a National Institutes of Health Career Investigator Award (K01 AI75089 to P.M.P.), and the National Institutes of Health (NIAID-R21-AI081164 to A.M.S. and P.M.P.).
Potential conflicts of interest. All authors report no conflicts of interest relevant to this article.

Christopher S. Hlady, MS; Monica A. Severson, MS; Alberto M. Segre, PhD; Philip M. Polgreen, MD, MPH

From the Departments of Computer Science (C.S.H., A.M.S.) and Industrial Engineering (M.A.S.), the Carver College of Medicine (P.M.P.), and the College of Public Health (P.M.P.), The University of Iowa, Iowa City, Iowa.

Address reprint requests to Philip M. Polgreen, MD, MPH, Division of Infectious Diseases, Department of Internal Medicine, The University of 
Iowa, Carver College of Medicine, 200 Hawkins Drive, Iowa City, IA 52242 (philip-polgreen@uiowa.edu).

Infect Control Hosp Epidemiol 2010; 31(9):975-977

(C) 2010 by The Society for Healthcare Epidemiology of America. All rights reserved. 0899-823X/2010/3109-0018\$15.00. DOI: $10.1086 / 655834$

\section{REFERENCES}

1. Boyce JM. Hand hygiene compliance monitoring: current perspectives from the USA. J Hosp Infect 2008;70(suppl 1):2-7.

2. Vigil C, Fornof M, Eckert B, Slotwinski L. Technology replaces paper trail tallying in hand hygiene compliance monitor. In: Program and abstracts of the First Annual Scientific Meeting of the Society for Heathcare Epidemiology of America (San Diego). 2009. Abstract 121.

3. Kerris N, Neumayr T. Apple's App Store Downloads Top Two Billion. Apple, 2009. http://www.apple.com/pr/library/2009/09/28appstore.html. Accessed January 12, 2010.

4. Eckmanns T, Bessert J, Behnke M, Gastmeier P, Ruden H. Compliance with antiseptic hand rub use in intensive care units: the Hawthorne effect. Infect Control Hosp Epidemiol 2006;27:931-934.

5. Pittet D, Allegranzi B, Boyce J, World Health Organization World Alliance for Patient Safety First Global Patient Safety Challenge Core Group of Experts. The World Health Organization guidelines on hand hygiene in health care and their consensus recommendations. Infect Control Hosp Epidemiol 2009;30:611-622.

\section{Importance of Alcohol in Skin Preparation Protocols}

To the Editor-The continued pursuit of lowering the risk of surgical site infection (SSI) has recently focused more attention on skin preparation solutions. Traditionally, no solution or technique for skin preparation has been widely held as superior to any other for preventing SSI after major operating room procedures. In the January 7,2010 , issue of The New England Journal of Medicine, Darouiche et $\mathrm{al}^{1}$ report a lower incidence of SSI associated with clean-contaminated surgical procedures among patients prepared with chlorhexidine plus alcohol, compared with the corresponding incidence among patients prepared with povidone-iodine (without alcohol), in a well-done, tightly controlled clinical trial. Much excitement has been generated by these results.

Our group recently reported a large, quasi-experimental study in Infection Control and Hospital Epidemiology ${ }^{2}$ in which we noted seemingly contradictory results, implying that a chlorhexidine-alcohol preparation was inferior to iodophorbased comparators. In contrast to Darouiche et al, ${ }^{1}$ however, our study uniformly used alcohol as an adjunct to iodophor preparations and identified a lower SSI rate in the iodophoralcohol preparation groups. Table 1 presents a side-by-side comparison of the results of these 2 studies.

Of note, the only 2 directly comparable groups (the chlorhexidine-alcohol groups) had very similar SSI rates of $9.5 \%$ and $10.1 \%$, suggesting relatively similar patient populations.
TA B LE 1. Surgical Site Infection (SSI) Rates (All Types) after Clean-Contaminated Surgical Procedures

\begin{tabular}{lc}
\hline Study, solution & $\begin{array}{c}\text { Rate of SSI, } \\
\text { proportion (\%) }\end{array}$ \\
\hline $\begin{array}{l}\text { Darouiche et al }{ }^{1} \\
\quad \text { Povidone-iodine (without alcohol) }\end{array}$ & $71 / 440(16.1)$ \\
$\quad$ Chlorhexidine-alcohol & $39 / 409(9.5)$ \\
Swenson et al $^{2}$ & \\
$\quad$ Povidone-iodine-alcohol & $44 / 541(8.1)$ \\
$\quad$ Iodine povacrylex-alcohol & $27 / 414(6.5)$ \\
$\quad$ Chlorhexidine-alcohol & $46 / 454(10.1)$ \\
\hline
\end{tabular}

Although the difference in protocols might seem minor, the rapid bactericidal activity of alcohol may be a vital part of any iodine-based skin preparation. ${ }^{3}$ The inclusion of alcohol in only 1 treatment arm in the study by Darouiche et $\mathrm{al}^{1}$ weakens the applicability of this otherwise excellent study.

We agree with Darouiche et al $^{1}$ that the practice of using iodophors alone to prepare the skin for an operation is inferior to use of a chlorhexidine-alcohol solution and that the practice should be abandoned. However, we also believe that the question of preoperative skin preparation solution superiority cannot be completely answered without an adequate experimental comparison of chlorhexidine-alcohol to iodophor protocols that also include the critical bactericidal activity of alcohol.

\section{ACKNOWLEDGMENTS}

Potential conflicts of interest. B.R.S. and R.G.S. received an unrestricted educational grant from $3 \mathrm{M}$.

Brian R. Swenson, MD, MS; Robert G. Sawyer, MD

From the Departments of Surgery (B.R.S., R.G.S.) and Public Health Sciences (R.G.S.), University of Virginia Health System, Charlottesville, Virginia

Address reprint requests to Brian R. Swenson, MD, MS, Department of Surgery, University of Virginia Health System, PO Box 800300, Charlottesville, VA 22908-0300 (brs3j@virginia.edu).

Infect Control Hosp Epidemiol 2010; 31(9):977-978

(C) 2010 by The Society for Healthcare Epidemiology of America. All rights reserved. 0899-823X/2010/3109-0019\$15.00. DOI: 10.1086/655843

\section{REFERENCES}

1. Darouiche RO, Wall MJJ, Itani KM, et al. Chlorhexidine-alcohol versus povidone-iodine for surgical-site antisepsis. $N$ Engl J Med 2010;362:1826.

2. Swenson BR, Hedrick TL, Metzger R, Bonatti H, Pruett TL, Sawyer RG. Effects of preoperative skin preparation on postoperative wound infection rates: a prospective study of 3 skin preparation protocols. Infect Control Hosp Epidemiol 2009;30:964-971.

3. Art G. Combination povidone-iodine and alcohol formulations more effective, more convenient versus formulations containing either iodine or alcohol alone: a review of the literature. J Infus Nurs 2005;28:314-320. 